\title{
Angular Dispersion Compensation Scheme for the Idler of Broadband Optical Parametric Amplifiers
}

\author{
Hüseyin Çankaya ${ }^{1,2, *}$, Giovanni Cirmi ${ }^{1,2}$, Peter Krogen ${ }^{3}$, Anne-Laure Calendron ${ }^{1,2}$, Yi Hua ${ }^{1}$ \\ Benoit Debord $^{4}$, Frédéric Gérôme ${ }^{4}$, Fetah Benabid ${ }^{4}$ and Franz X. Kärtner ${ }^{1,2}$ \\ ${ }^{I}$ Center for Free Electron Laser Science (CFEL), Deutsches Elektronen-Synchrotron (DESY) \& Department of Physics, \\ University of Hamburg, Notkestrasse 85, 22607 Hamburg, Germany \\ ${ }^{2}$ The Hamburg Center for Ultrafast Imaging, Luruper Chaussee 149, 22761 Hamburg, Germany \\ ${ }^{3}$ Department of Electrical Engineering and Computer Science and Research Laboratory of Electronics, \\ Massachusetts Institute of Technology (MIT), Cambridge, Massachusetts 02139, USA \\ ${ }^{4}$ GPPMM Group, Xlim Research Institute, UMR 7252 CNRS, University of Limoges, Limoges, France \\ *huseyin.cankaya@cfel.de
}

\begin{abstract}
We demonstrate a technique for compensation of the idler angular chirp from an optical parametric amplifier in non-collinear geometry. This method enables generating broadband CEPstable pulses for seeding high-energy optical parametric amplifiers.
\end{abstract}

There is an increasing demand for carrier envelope phase (CEP) stable, few cycle, high-energy pulses in the near and mid-infrared spectral regions for applications such as high-harmonics generation, strong-field physics and ultrafast spectroscopy. Optical parametric amplifiers (OPAs) and optical parametric chirped pulse amplifiers (OPCPAs) are strong candidates for the generation of such pulses. Those amplifiers provide ultra-broad spectral bandwidths supporting few or single cycle transform-limited pulses. In addition, OPAs and OPCPAs provide passively CEP stable pulses in the idler beam when seed and pump pulses have the same CEP even though the CEP of the driver laser is not stabilized [1-3]. Type I phase matching typically provides the broadest spectrum for those amplifiers. In noncollinear geometry, the idler is angularly dispersed which limits its applications. Several methods have been proposed to compensate the angular dispersion of an idler beam of an OPA and OPCPA via gratings or prisms [4-6]. Here, we use coupling into a hollow-core fiber to eliminate angular dispersion. As a proof of principle experiment, we coupled the angularly dispersed idler beam from an OPA producing pulses at $2 \mu \mathrm{m}$ with transformlimited pulse duration of 24 fs into a hollow core photonic crystal fiber (HCPCF). In particular, we used a Kagome fiber in the linear regime, since it can handle pulses with tens of $\mu \mathrm{J}$ pulse energies without introducing nonlinearity.

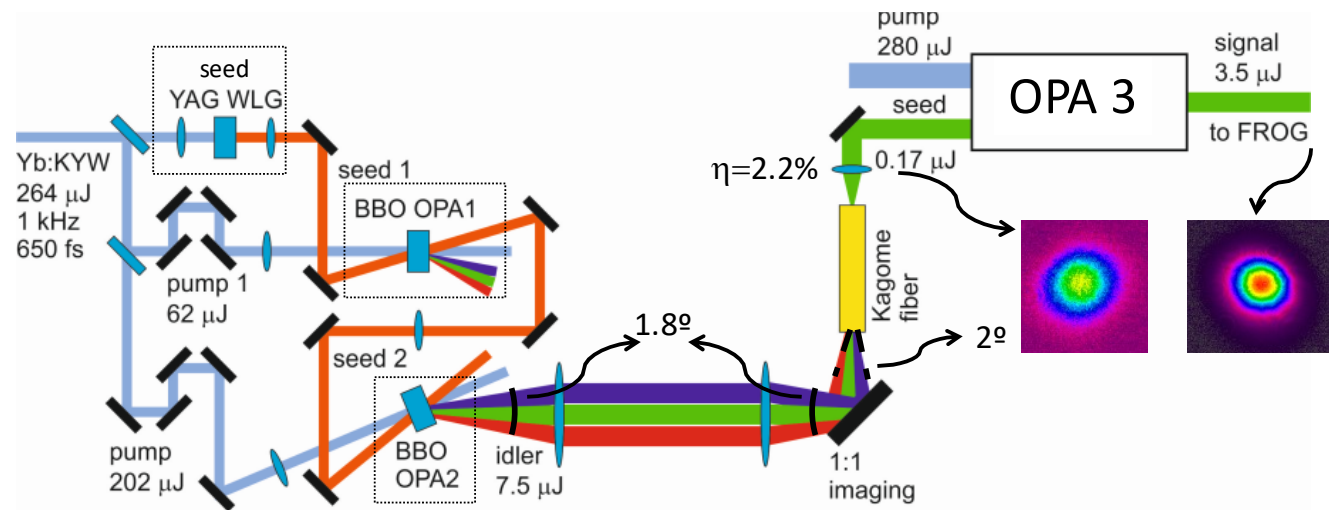

Figure 1: Schematic of the broadband CEP-stable seed generation from angularly dispersed idler, near field beam profile after Kagome fiber and third optical parametric amplifier (OPA3).

Figure 1 shows the setup of the broadband CEP-stable seed generation from the angularly dispersed idler, the near field beam profile after the Kagome fiber and the third optical parametric amplifier (OPA3). We utilized 264- $\mu \mathrm{J}$ pulses at $1 \mathrm{kHz}$ from a home-made $\mathrm{Yb}: \mathrm{KYW}$ regenerative amplifier for generating seed pulses via white-light continuum generation in 10-mm YAG and two consecutive OPA stages based on type I phase matching configuration in $\beta$-barium borate (BBO) nonlinear crystal. The first (OPA1) and the second (OPA2) amplification 
stages were pumped by pulses with $62 \mu \mathrm{J}$ and $202 \mu \mathrm{J}$ energies, respectively. The angularly dispersed idler generated in OPA2 was imaged at the entrance of the Kagome fiber with acceptance angle of $2^{\circ}$. The angular dispersion of the idler beam was measured to be $1.8^{\circ}$. The output of the Kagome fiber was collimated by using a converging lens. We scanned the near-field beam profile via a fiber coupled spectrometer and we did not observe any spatial chirp residual due to angular chirp from OPA2. The collimated output of the Kagome fiber was used as a seed source for OPA3, pumped by $280-\mu \mathrm{J}$ pulses where the $0.17 \mu \mathrm{J}$ input pulses are amplified to $3.5 \mu \mathrm{J}$.
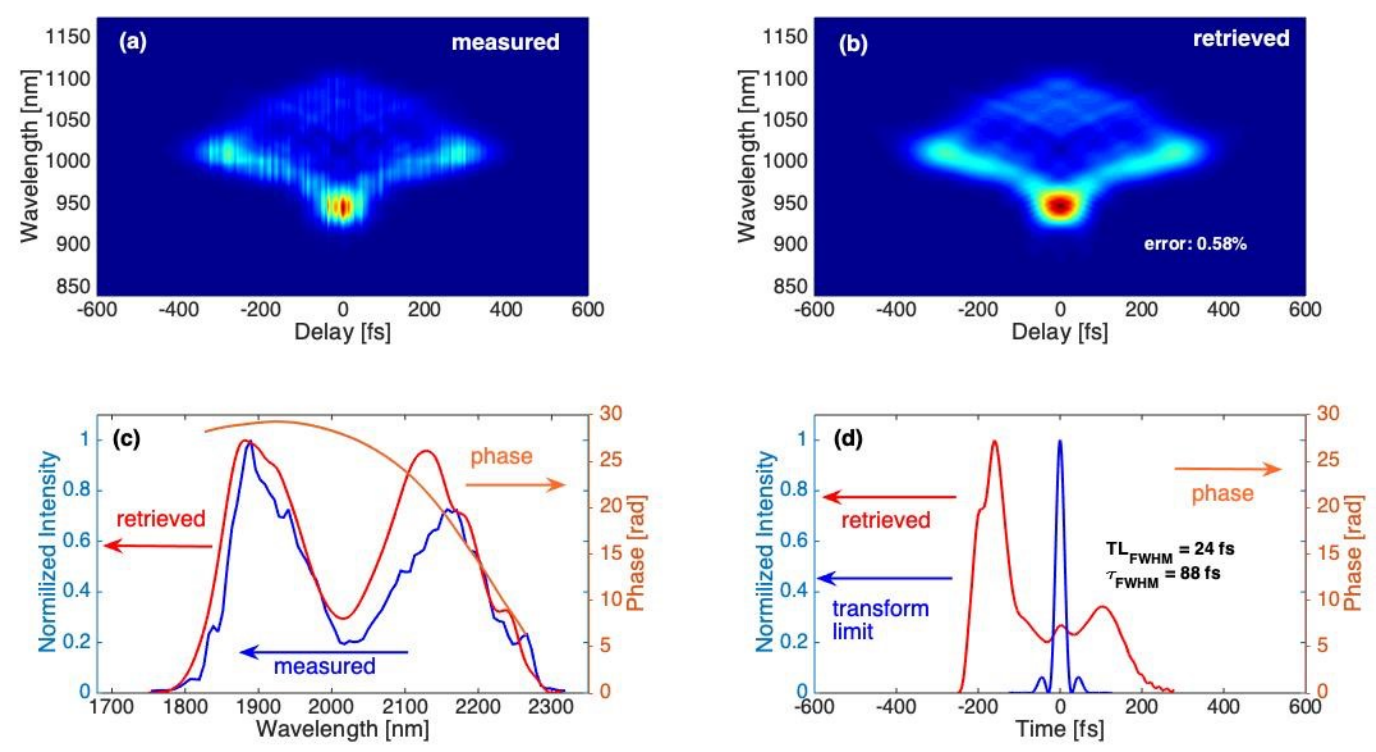

Figure 2: Measured (a) and retrieved (b) second harmonics FROG traces of the broadband third OPA stage; (c) measured (blue) and retrieved (green) spectral intensity, retrieved spectral phase (orange); (d) transform limit of the measured spectrum (blue), retrieved temporal intensity (green) and temporal phase (orange). The reconstruction error is $0.58 \%$.

The pulses at the output of the OPA3 are characterized by using second harmonics generation frequency resolved optical gating (FROG) technique. Figure 2 (a) and (b) show the measured and retrieved FROG traces, respectively, where the FROG reconstruction error is $0.58 \%$. As can be seen in Figure 2 (c), the measured and the retrieved spectrum obtained from the FROG algorithm match well and the spectral phase has a good behavior which can be compensated with standard techniques. The pulse duration (Figure 2 (d)) was determined to be $88 \mathrm{fs}$ and the transform-limit was $24 \mathrm{fs}$.

In summary, we demonstrated an elegant technique for compensating the angular -chirp of the idler from an optical parametric amplifier in non-collinear geometry. This method enabled the generation of broadband CEPstable pulses centered around $2 \mu \mathrm{m}$ for seeding high energy optical parametric amplifiers.

\section{References:}

[1] A. Baltuška, T. Fuji, and T. Kobayashi, "Controlling the Carrier-Envelope Phase of Ultrashort Light Pulses with Optical Parametric Amplifiers" Phys. Rev. Lett. 88, 133901 (2002).

[2] C. Manzoni et al., "Tunable few-omptical-cycle pulses with passive carrier-envelope phase stabilization from an optical parametric amplifier", Appl. Phys. Lett. 90, 171111 (2007).

[3] G. Cirmi et al., "Carrier-envelope phase stable, few-optical-cycle pulses tunable from visible to near IR", JOSA B 25, B62 (2008).

[4] A. Shirakawa, et al., "Pulse-front-matched optical parametric amplification for sub-10-fs pulse generation tunable in the visible and near infrared", Opt. Lett. 23, 1292 (1998).

[5] K. Yamane, et al., "Angularly-dispersed optical parametric amplification of optical pulses with one-octave bandwidth toward monocycle regime”, Opt. Exp. 16, 18345 (2008).

[6] A. Okamura, et al., "Octave-spanning carrier-envelope phase stabilized visible pulse with sub-3-fs pulse duration", Opt. Lett. 36, 226 (2011). 